\title{
ENVIRONMENTAL EFFECTS ON THE PROPERTIES OF \\ SPIRAL GALAXIES: ISOLATED PAIRS OF SPIRALS
}

\author{
I. MÁRQUEZ \\ Institut d'Astrophysique de Paris \\ 98 bis, Bd Arago, 75014 Paris (France)
}

We characterized isolated spiral galaxies as a reference for the properties of interacting ones: we selected all the spirals in CfA catalog with $\mathrm{m}_{B} \leq 13.0, \delta \geq 0, \mathrm{a} \leq 4^{\prime}$ and $73^{\circ}>i>32^{\circ}$. The isolated ones were those having no companions in $\Delta \mathrm{D} \leq 0.5 \mathrm{Mpc}$ and $\Delta \mathrm{V} \leq 500 \mathrm{~km} / \mathrm{s}$ and no satellites galaxies in their neighborhood (from Nilson catalog and Palomar charts). To select the isolated pairs of spirals we used Karachentsev's catalog applying similar conditions for $\mathrm{m}_{B}, \delta, a, i$ and the same isolation criteria. We used broad band CCD images (Johnson B,V e I), narrow band CCD images $(\mathrm{H} \alpha)$ and long slit spectra in $\mathrm{H} \alpha$ region. The BVI images analysis consisted on applying sharp-divided methods, obtaining simulated images (from bulge/disk decomposition and galaxy orientation in the sky) and Fourier analysis. From $\mathrm{H} \alpha$ images we determined total emission, size, distribution and flux of HII regions. We also obtained the rotation curves.

Isolated Galaxies: they present uniform disks, smooth color gradients and flat rotation curves. From the Principal Component Analysis applied to the analyzed properties we find that two eigen-vectors explain the $95 \%$ : (1) Mass, luminosity, size or specific angular momentum; (2) B/D or G (inner gradient of the rotation curve).

Isolated Pairs: they show distorted morphologies (warps, $\mathrm{X}$ isophotes, prominent outer rings, tails, plumes, bridges). Interacting spirals are more luminous, with higher surface brightness, bluer total colors (greater dispersions) with stronger color gradients and redder central colors. Star forming processes are more efficient. Some of the interacting galaxies show TYPE II PROFILES (but not even an isolated one). Some of the interacting galaxies show declining rotation curves. These results are explained as due to the mass redistribution provoked by the interaction, which implies local inestabilities and mass transport to the center (Márquez, I., 1994, PhD Thesis, Universidad de Granada, Spain, and references therein). 